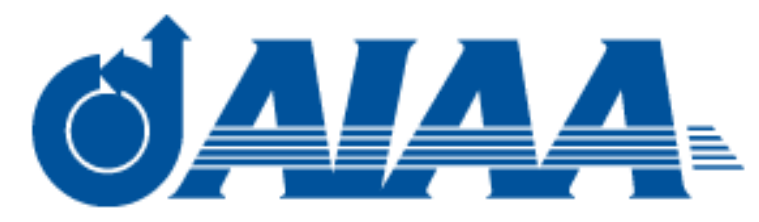

\title{
Ant-based automated mission planning for Data Relay space missions
}

\begin{tabular}{|r|l|}
\hline Journal: & Journal of Aerospace Information Systems \\
\hline Manuscript ID & $2018-09-$-I010689.R1 \\
\hline Manuscript Type: & Full Paper \\
\hline Author: & 15 -Jan-2019 \\
\hline Complete List of Authors: & $\begin{array}{l}\text { Ntagiou, Evridiki; University of Surrey, } \\
\text { Armellin, Roberto; University of Surrey, Surrey Space Centre } \\
\text { Iacopino, Claudio; Surrey Satellite Technology Ltd } \\
\text { Policella, Nicola; European Space Agency, } \\
\text { Donati, Alessandro; European Space Operations Centre }\end{array}$ \\
\hline Subject Index Category: & $\begin{array}{l}\text { 12110 Artificial Intelligence Systems < 12000 COMPUTING, } \\
\text { INFORMATION, AND COMMUNICATION, 12300 Autonomous Systems < } \\
\text { 12000 COMPUTING, INFORMATION, AND COMMUNICATION, 14400 } \\
\text { Software Systems < 14000 REAL-TIME SYSTEMS }\end{array}$ \\
\hline & \\
\hline
\end{tabular}

\section{SCHOLARONE Manuscripts}




\title{
Ant-based automated mission planning for Data Relay space missions
}

\author{
Evridiki V. Ntagiou* and Roberto Armellin ${ }^{\dagger}$ \\ Surrey Space Centre, University of Surrey, Guildford, GU2 7XH, United Kingdom \\ Claudio Iacopino \\ Surrey Satellite Technology, Guildford, GU2 7YE, United Kingdom \\ Nicola Policella ${ }^{\S}$ and Alessandro Donati ${ }^{\text {Il }}$ \\ ESOC, European Space Agency, Robert-Bosch-Strasse 5, 64293 Darmstadt, Germany
}

The Earth Observation (EO) market is rapidly growing due to technology miniaturization, cheaper launch opportunities and wider spectrum of EO applications. Along with an exponential growth of the ground based users that can access Low Earth Orbit (LEO) spacecraft data, this growing community represents an important demand for Data Relay missions. LEO spacecraft have short visibility windows to the Ground Stations (GS) which limit their throughput. Data Relay missions are comprised of spacecraft at higher altitude orbits (Geostationary Orbits) acting as relays of data among LEO spacecraft and GS. Those missions are then able to offer more frequent data downlink opportunities to the LEO spacecraft thus increasing the volume of the data reaching the ground and improving the responsiveness between users' requests and downlink operations. Ground based Mission Planning Systems (MPS) are commonly managing such complex missions, representing a large operational cost. In this paper, we propose the application of a Swarm Intelligence algorithm to the design of an automated MPS for Data Relay missions. Automated MPS have the potential of saving operational costs while leaving the high level decisions to human operators. This paper represents the first time that an Ant Colony Optimization (ACO) algorithm is applied to this type of scheduling problem. This family of algorithms is generally found to offer good level of efficiency and scalability. In this work, we compare our approach against an algorithm popular in literature, called Squeaky Wheel Optimization (SWO) and show how our ACO algorithm outperforms it.

\footnotetext{
*PhD Student, Surrey Space Centre, e.ntagiou@surrey.ac.uk

$\dagger$ University Lecturer, Surrey Space Centre, r.armellin@surrey.ac.uk

$\doteqdot$ Mission Planning Engineer, Ground Software Team, c.iacopino@sstl.co.uk

$\S$ Senior Research Engineer, Advanced Mission Concepts and Technologies, nicola.policella@esa.int

IISection Head, Advanced Mission Concepts and Technologies, alessandro.donati@esa.int
} 


\section{Introduction}

M ISSIONS observing and monitoring the Earth have received an increasing amount of attention due to the importance of the data they provide users with, ranging from weather forecast and science applications to Earth imaging data. Thus, both the population of Earth Observation (EO) spacecraft and users that can access their data have increased, resulting in a corresponding increase of the amount of data produced on board. This data needs to be transmitted to the ground as soon as possible in order to free on board resources, or satisfy urgent user requests. To that respect, data relay missions are being designed and operated. In those missions, spacecraft placed in higher altitude orbits, e.g. $36000 \mathrm{~km}$, called Geostationary (GEO) orbits, act as relays of data among spacecraft on lower altitude orbits, e.g. $600 \mathrm{~km}$, called Low Earth Orbits (LEO), and Ground Stations (GS). GEO spacecraft are permanently fixed in the same position of the sky with respect to the Earth and have a very wide field of view on the Earth's surface and constant visibility to a number of Ground Stations. LEO spacecraft on the other hand, can have higher resolution, smaller and cheaper platforms, less expensive launch process, but they have short visibility windows with the Ground Stations thus their throughput is limited by their downlink opportunities. In data relay missions, LEO spacecraft data can be sent to GEO spacecraft and then be transmitted to a Ground Station, reducing the time delays in the communication among LEO and GS almost regardless of their relative position. The Tracking Data Relay Satellites System (TDRSS) [1] from the National Aeronautics and Space Administration (NASA) and the European Data Relay System (EDRS) [2] from the European Space Agency (ESA) with 10 and 1 operating GEO spacecraft respectively, are two examples of such missions.

Planning and Scheduling for a data relay mission is the process of determining whether and when each LEO spacecraft will be assigned a time slot to communicate with the GEO satellite. A space Mission Planning System (MPS) is a ground based software system that produces feasible mission schedules taking into account the user requests and the mission capabilities and constraints. There can be dozens or even hundreds of LEO spacecraft submitting communication requests, but the lack of available resources might only allow for a subset of them to be accommodated. This is very common in the Earth Observation market, due to the increasing number of simultaneous requests by the users but no sufficient resource availability. Scheduling problems in which the initial set of tasks is larger than the set of those that can be scheduled are called oversubscribed. In the specific mission planning problem, the requests can also be flexible i.e. they can be scheduled within a given time window that is longer than their actual duration, increasing the complexity of the planning process significantly. Given this problem complexity and with spacecraft being expensive resources with a costly construction process and limited lifetime, modern MPS employ optimization algorithms to guarantee that satellites will be cautiously and optimally managed [3][4][5]. The performance of such systems cannot only be measured by their ability to optimize an objective though. In a highly dynamical environment like space, other performance metrics can be the system's efficiency and scalability, as we aim for a system that responds quickly and provides good solutions regardless of the workload.

In the literature, oversubscribed scheduling problems are solved with heuristics due to their extremely large size; 


\section{Relevant Work}

In this section, we discuss the existing literature on the scheduling problems ACO has been used for and the current heuristic approaches in solving oversubscribed scheduling problems. We discuss a critical characteristic for the performance of heuristics, which is the balance between exploration and exploitation phases and conclude with the novelties of this work on the aforementioned fields.

Ant colony optimization algorithms have been applied to a wide range of scheduling problems $[8]$ and several engineering domains [9]. There are only a few applications that resemble the planning problem we address in this paper that have applied an ACO algorithm to solve it. In [10], the goal is to schedule a set of flexible activities to a number of resources so that the makespan of the schedule is minimized. The resources considered are non binary i.e. two activities can be scheduled at the same time so long as they do not exceed the given capacity constraints at any time. The problems considered are not oversubscribed, but the search focuses on finding the optimal schedule which includes all the tasks. Tests were performed on problems with 120 activities to be scheduled on 4 resources. Compared 
to several other methods including genetic algorithms (GA) and simulated annealing (SA), the proposed ACO algorithm performed best on average. In [11] the authors address a job shop scheduling problem [12] of small size, with 3 jobs to be scheduled in 3 machines. The jobs are flexible with relaxed earliest and latest delivery times which, if violated, induce costs; the goal is to maintain those costs at minimum levels.

Oversubscribed scheduling problems appear in the literature with different constraints and objectives; what is common is the inability to accommodate all the tasks in a single solution, unless the tasks' characteristics are adjusted. In one group of algorithms that is widely used in the space industry, an initial solution is produced greedily based on a criterion e.g. task priority, and is then improved. One example of such a repair based algorithm is presented in [13] where the USAF Air Mobility Command problem is solved. Flexible tasks of fixed duration need to be scheduled on non binary resources. The problem is solved with an iterative repair search based algorithm in which the improvement mechanism is based on swaps and a new heuristic based on the maximum flexibility is introduced. The latest trend in space mission planning and scheduling includes a similar method that involves randomness in producing the initial solutions which are then also improved, called Squeaky Wheel Optimization (SWO) [14] [7] [15] [16]. In this method, the improvements are choices of changes in the order by which the tasks are considered for scheduling. In [17], the Air Force Satellite Control Network (AFSCN) problem, with flexible tasks with preferences to the available binary resources, is solved using SWO. The method was found to produce good solutions in few iterations ( 4000 for 500 tasks within one day), though it was outperformed by a genetic algorithm once more iterations $(\sim 8000)$ were considered. In [15], SWO is used to solve the oversubscribed scheduling problem of Deep Space Network, with tasks of little flexibility and a goal to maximize the overall scheduled time for all missions. The most promising results are obtained with a variant of SWO combined with a rule that limits each mission's input based on reduced input levels the users have accepted in the past, as a tactic to make the problem less oversubscribed.

All the afore mentioned heuristic algorithms employ a fixed exploration/exploitation balance. During an exploration phase the search is directed towards unexplored areas of the search space, in order to acquire a better view of its shape and size. On the other hand, during an exploitation phase the search is directed towards regions of the search space that include the best solutions found so far, so as to fine tune them by searching close to them. As stated in [18], "Exploitation and exploration are opposing forces that need to be balanced". Balancing exploration and exploitation phases is the equivalent to balancing having big and small changes' set size when improving a solution in SWO algorithms; this characteristic is found to be a critical factor for their performance [17].

In this paper, we chose to study the application of an ACO algorithm to an oversubscribed problem of flexible tasks that request to be scheduled in a binary resource, within a finite time window. The proposed ACO algorithm modifies the exploration/exploitation balance while the algorithm runs. We use a mechanism to change this balance that was proposed in [19] for binary chain graphs, but apply it to directed graphs that are not constrained in their form, adding a heuristic function to better guide the search. ACO and SWO are inherently different with respect to how their 


\section{Problem definition}

In this section, we discuss the oversubscribed scheduling problem that occurs in data relay space missions. Details that are not relevant to the planning and scheduling process have been omitted. In the problem we consider, $G$ GEO spacecraft act as relays, and $N$ LEO spacecraft users submit requests for communication. Each LEO request can be accommodated by all or by a subset of the $G$ GEO satellites but will only be scheduled in one. An example of a simplified configuration considering 3 LEO and 1 GEO spacecraft is shown in Fig. 11. For the rest of the paper, we will use the term link when referring to the communication requests. Each link is associated with the following values:

- Earliest start time, e.s.t, for each of the GEO spacecraft it can be accommodated by

- Latest end time, l.e.t, for each of the GEO spacecraft it can be accommodated by

- Duration, dur

- Priority, $p$

The number of GEO resources a link can be accommodated by is a randomly chosen value between 1 and $G$. The time window l.e.t. - e.s.t. is called feasibility window. All values are positive and fixed for each link $i$, with $d u r_{i} \leq$ l.e. $t_{i}-$ e.s.t $t_{i}$. A priority value $p_{i}$ is associated with each single link and can reflect different characteristics, from the order of arrival to the link's cost. The planning horizon is the time window under consideration in which the plan will be executed. All the submitted links should start and end within it. Thus, if the planning horizon is the time window $[l, h]$, then $\min \left\{\right.$ e.s.t $\left.t_{i}\right\} \geq l$ and $\max \left\{l\right.$.e. $\left.t_{i}\right\} \leq h$ for all links $i$. A typical duration of the planning horizon is 1 day. The planning process that regards this planning horizon is completed a given amount of time before the lower bound of it, $l$, to allow for the solution plan to be uploaded to the spacecraft. In this paper, only the links that have been submitted prior to the beginning of the planning process are considered for scheduling.

\section{A. Problem Constraints}

1) For every link, its e.s.t. and l.e.t. cannot be smaller or larger than the lower bound and upper bound of the planning horizon respectively.

2) No link can start before the earliest start time, or finish after the latest end time. If $t_{s}$ is the actual start time of a link, then e.s.t $\leq t_{s} \leq$ l.e.t - dur.

3) The link requests cannot be split into several parts. 


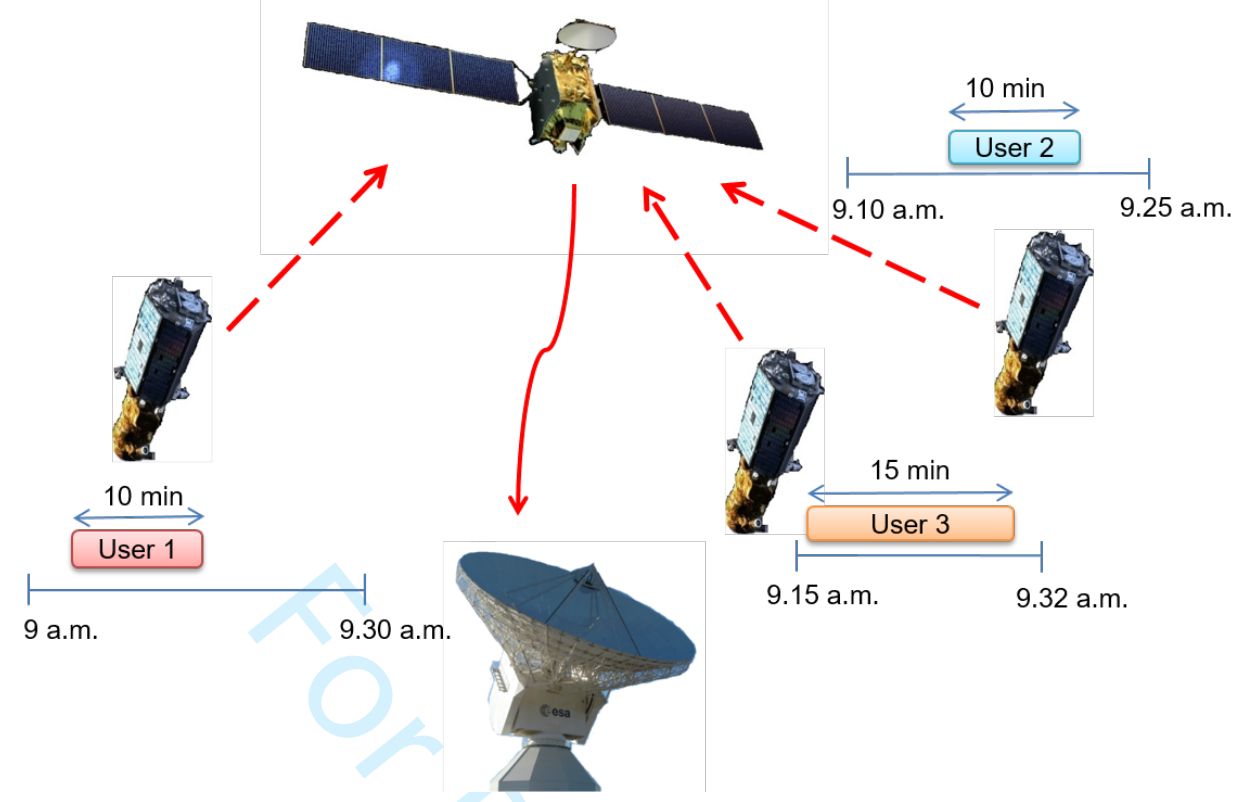

Fig. 1 Data relay space mission configuration with 3 LEO and 1 GEO spacecraft

4) Two links cannot be accommodated at the same time; a gap of $k_{i j}$ time units is needed between two consecutive scheduled links $i$ and $j$. The value of $k_{i j}$ depends on the links' $i$ and $j$ characteristics. The GEO resource is binary, thus for the actual start time $t_{s}$ of all $i, j$ links where $i \neq j$, and taking into account constraint 3 , we have either $t_{s i}+d u r_{i}+k_{i j} \leq t_{s j}$ or $t_{s j}+d u r_{j}+k_{i j} \leq t_{s i}$

5) Each link will only be scheduled in a single GEO satellite.

The individual requests are feasible i.e. both LEO spacecraft and the GS they want to transmit their data to, are within the field of view of the GEO satellite during the whole feasibility window. The MPS will produce schedules that satisfy the constraints and optimize a given objective function $f$. In this paper, we consider 2 different objective functions, presented in Sect. III.B.

Data relay missions have idle time windows, or time windows in which a GEO resource cannot accommodate any communication requests, due to e.g. housekeeping services. Those time windows are communicated to the users before their links' details are submitted; in case of feasibility windows' overlap with the idle time windows, the users are notified accordingly and asked to resubmit their requests with revised feasibility windows. Given that the GEO resources are binary, one idle time window breaks a planning horizon to two shorter resulting in two separate planning problems. Hence, we do not consider idle time windows in this paper. 


\section{B. Objective functions}

We have chosen to investigate two different mission objectives; in this way, we show how the solving approach is disconnected from the objective for both ACO and SWO, so long as the objective function is a combination of some of the links' characteristics e.g. priority, duration e.t.c.

\section{Maximizing the number of scheduled Links}

The first objective is to maximize the number of scheduled links. A data relay service provider is expected to want to accommodate as many user requests as possible. In this case, the objective function is formulated as

$$
f=\frac{\operatorname{card}\{\mathcal{S}\}}{\operatorname{card}\{\mathcal{P}\}}=\frac{\text { Number of links scheduled in all the GEO spacecraft }}{\text { Total number of links }}
$$

where $\mathcal{S}$ is a feasible schedule and $\mathcal{P}$ is the initial set of all the links. The planning horizon is finite, thus in order to accommodate as many links as possible, a preference is given to the shorter ones. The heuristic function, $\eta$ in Eq. (5), will be equal to :

$$
\eta_{i j}=\frac{1}{d u r_{j}}
$$

for arc $i j$, where $d u r_{j}$ is the duration of link $j$. Thus, the ACO transition rule from one node to another (5) will favour the shorter links. This function is also used as a trouble making measure in SWO, where the higher the value of (2) for an unscheduled link, the more moves forward it does. The index $i$ does not have any role for SWO.

\section{Maximizing the priorities}

The second objective is the maximization of the sum of the scheduled links priorities. This objective can easily be linked to the maximization of the data relay service provider's profit. This is the case if the priority is proportional to the link cost which can, in turn, be dictated by the demand for communication during the link's feasibility window. The priority value can also reflect the urgency of certain links to be scheduled, in the case of an emergency, e.t.c. It is worth noting that the priority is not necessarily proportional to the link duration; a shorter link can end up having higher priority than a longer link.

The objective function is formulated:

$$
f=\frac{\sum_{i \in \mathcal{S}} p_{i}}{\sum_{i \in \mathcal{P}} p_{i}}
$$

where $\mathcal{S}=\{j, k, \ldots, w\}$ is the union of all GEO spacecraft' feasible schedules, $j, k, w$ are links and $p_{i}$ is the priority value of link $i$. Since the planning horizon is finite, the maximization of (3) is indirectly associated with the duration of the scheduled links. In order to maximise the objective function we want to have, at any instant, the maximum priority/duration ratio such that the integral over the finite window is maximized. The heuristic function $\eta$ in $(5)$ is thus 
defined as:

$$
\eta_{i j}=\frac{p_{j}}{d u r_{j}}
$$

where $i$ in the origin node of arc $i j$ on an ACO graph described in Sect. IV.B $j$ is the destination node and $d u r_{j}$ is the duration of link $j$. In SWO, the higher the value of (4) for an unscheduled link, the more moves forward are assigned to it. The index $i$ does not have a role in SWO.

\section{Solving approach}

\section{A. Ant Colony Optimization}

Ant Colony Optimization (ACO) is a probabilistic method used in finding solutions to Computer Science and Operations' problems that can be reduced to finding optimal paths in graphs [6]. Real world ant colonies are able to find the shortest paths between their nests and a food source, using no direct communication with each other. When searching for food the ants first wander randomly, but upon finding a source of food, they return to their colony laying down pheromone in the path they followed. If other ants sense such a trail, they are likely to follow it and in case it leads them to food, they will also reinforce it with pheromone. The trails evaporate over time, making the total amount of pheromone in shorter paths higher, since they get marched over more frequently. From a more abstract point of view, we see that none of the individual ants has universal knowledge of the colony's actions, and they all follow simple sets of rules. Nevertheless, the colony presents a complex behavior, which is the result of the ants' interaction with the environment. This mechanism is called stigmergy, a means of indirect coordination of a number of individuals, through their environment. The basic principle of stigmergy is that the traces left in the environment by one individual's actions stimulate the actions of the next.

In order to apply an Ant Colony Optimization algorithm to an optimization problem, the problem environment must be translated to a graph. A graph $G=(V, E)$ consists of a set $V$ of vertices (or nodes), and a set $E$ of undirected edges or directed arcs connecting nodes. An arc can have a weight representing a problem entity. A path is a set of arcs with the same direction, connecting some or all the nodes of the graph. A typical ACO algorithm involves three main steps:

1) Path Construction: The ants traverse the graph and construct a path. Starting from an initial node they iteratively add an arc to the path, choosing among their options based on the amount of pheromone. The probabilistic rule they use to choose an arc favours the one with the highest pheromone amount:

$$
P_{i j}=\frac{\tau_{i, j}^{\alpha} \eta_{i, j}^{\beta}}{\sum_{j=1}^{M_{i}} \tau_{i, j}^{\alpha} \eta_{i, j}^{\beta}}
$$

where $\tau_{i, j}$ is the amount of pheromone in the arc connecting nodes $i$ and $j$ (or arc $i j$ ), $\eta_{i, j}$ is the value of the heuristic $\eta$ or, in other words, the weight of the same arc and $M_{i}$ is the number of incoming arcs in the node $i$. 


\section{B. Directed Weighted Graph Representation}

The problem representation can have a significant role in the performance of an algorithm. In this section, we propose a directed graph representation for the oversubscribed scheduling problem described in III, partly integrating the problem constraints. In this work, we build the graph taking into account the nature of the objectives under investigation which dictate that the more links included in a feasible path, the higher the objective function value. The directed graph consists of:

- $N$ nodes, each representing a link. From now on link and node will refer to the same thing.

- $E$ Arcs, each connecting 2 nodes, $i$ and $j$. If an arc is incoming to node $i$, link $i$ can succeed link $j$ in a schedule, and vice versa. More specifically, an arc from a node to another is added if the corresponding links have non-overlapping feasibility windows or despite their windows overlapping, both links can be scheduled. The requirements translate to:

- an arc from node $i$ to node $j(\operatorname{arc} i j)$ being added if

$$
\begin{aligned}
& \text { e.s.t.j } \geq \text { l.e.t.i } \text {, or } \\
& \text { e.s.t. } \cdot{ }+d u r_{i}+k+d u r_{j} \leq \text { l.e.t.j }
\end{aligned}
$$

- an arc from node $j$ to node $i$ (arc $j i)$ being added if

$$
\begin{aligned}
& \text { e.s.t. } i \geq \text { l.e.t.j, or } \\
& \text { e.s.t.j }+d u r_{j}+k+d u r_{i} \leq \text { l.e.t. } i
\end{aligned}
$$

Essentially, in this graph representation each pair of nodes that are connected by an arc forms a feasible combination of links. The graph representation for the data relay system configuration shown in Figure 1 can be found in Figure 2 
When more than one GEO spacecraft are considered, the links' duration values remain fixed, but the time window for each GEO resource is different, due to their different position with respect to the Earth. The resources, thus, 'view' the problem slightly differently from one another. To that respect, we build a different graph for each GEO resource considering only the links it can accommodate.

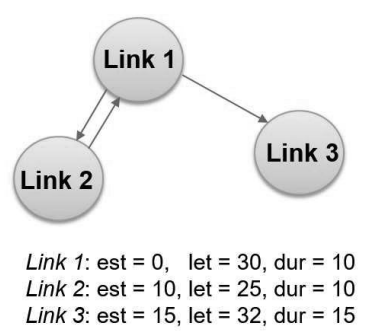

\section{Fig. 2 Graph representation for the data relay system configuration shown in Fig 1}

\section{Algorithm}

The algorithm we use follows the three main steps described above, including an additional one. The new step concerns a way to control the balance of exploration and exploitation phases [18]. In [19] it is proven that for problems that can be represented by binary chains, when the parameter $\alpha<1$ in Eq. (5), the exploration is higher than exploitation whereas the opposite happens when $\alpha>1$. In this paper, we apply this algorithm to the graphs described in Sect. IV.B. changing the balance of exploration and exploitation by changing the value of alpha while the algorithm runs and use a heuristic function $\eta$ to guide the search. In [20], the author presents results on an increased algorithm performance when an increasing $\alpha$ is used. After testing different increasing functions that combine the three different behaviours of the colony, like linear and exponential, we found that the function shown in Fig. 3. a tangent profile in the interval [0.5, 2] with the flex at $\alpha=1$, results in the best solutions. A high level workflow of the algorithm is shown in Algorithm 1

In the pheromoneFieldInitialization() function we assign the same amount of pheromone on all the arcs in the beginning of the run. The chooseFirst() function defines the node from where the search will begin for each spacecraft, as described in Sect. IV.C.1. We select an arc and proceed to the next node with arcSelection() which is based on the probabilistic rule (5). After a node is chosen, startTimeFix() fixes its start time to the earliest possible available time e.g. the start node will be scheduled on its e.s.t. Among all the outgoing arcs of a node, only the ones that lead to links that do not overlap with the current schedule are considered for selection. For example, if a schedule consists of links $\{4$; 6; $2 ; 9\}$ (link IDs) in a chronological order, and link 9 has an arc directed to link 1 , then if $\{4 ; 6 ; 2 ; 9 ; 1\}$ is not a feasible combination of links - even though $\{9 ; 1\}$ is a feasible combination - then this arc will not be considered in the weighted roulette mechanism for selection. If $e$ nd is the latest occupied time from link $i$, then we check which arcs lead to feasible combinations by checking if l.e.t.e $-d u r_{e}-k_{i e}>$ end for all outgoing arcs $(i, e)$. If this inequality holds, then arc 
$(i, e)$ is considered for selection. Every time an ant adds a link to the schedule, this choice is communicated to the rest of the spacecraft' via broadcast() method and the chosen link is removed from their graphs. The path construction continues for all spacecraft in parallel; one link at most is added to each resource's schedule before the next spacecraft in order. The order by which the GEO spacecraft add links is random. Each chosen link is scheduled at the end of the current schedule, before the next node that will be added is chosen. When the roulette is empty, the path construction is completed. The created path is evaluated by an objective function $f$ (pathsEvaluation()), based on which we update the pheromone field, by evaporating and depositing an amount of pheromone, in pheromoneFieldsUpdate(). In the alphaUpdate() function, the value of alpha increases as shown in Fig. 3 in order to increase the pheromone field effect in the ants' choice.

\section{Choosing the first node}

Given an objective that is not proportional to the time left idle in the GEO resource, the chronologically first link should begin close to the lower bound of the planning horizon. In order to not jeopardize the quality of the solution though, we do not assume that the first link should be the one with the smallest e.s.t. value, but we choose it among the ones with the earliest e.s.t. We use a weighted roulette that favours the link that adds more value to the objective function. Adding the earliest 3.5\% of the links to the weighted roulette and choosing the first node among them, is empirically found to produce better results for the problems considered in this work. It should be noted that the nodes considered as first nodes are not necessarily mutually exclusive with each other. 


\title{
2. Pruning
}

When constructing a path in the graph we run the risk of creating schedules with long periods of idle time. Even though the objective function dictates whether that is a desirable feature or not, we generally believe that a data relay mission cannot be considered successful if the GEO resource is left idle for long. In order to avoid even considering such schedules as candidate solutions, we do not add all the arcs that satisfy conditions (6) and (7). For two links $i$ and $j$, if

$$
\text { l.e.t. }{ }_{i} \leq \text { e.s.t.j } \text { and/or l.e.t.j } \leq \text { e.s.t. } i
$$

arcs $i j$ and/or $j i$ might not be added to the graph, in order for the ants to be directed to paths that consider links between $i$ and $j$, whenever that is possible. Even though the more oversubscribed a problem is, the more chances of finding a path between two links $i$ and $j$ we have, that is not always the case. We need to be certain that a path between $i$ and $j$ exists by finding at least 1 link that could be accommodated between them, otherwise not adding the arc $i j$ (or $j i$ ) may lead to non optimal results. In order to explain the process, we consider links $i$ and $j$ for which l.e.t. $i \leq$ e.s.t.j. We find the actual time distance between the two links' feasibility windows $d_{i j}=$ e.s.t $t_{j}-$ l.e. $t_{i}$. In an ascending order of the links based on their duration, we use binary search to find the ones with durations smaller than $d_{i j}$ and examine whether they fit between links $i$ and $j$, taking into account the time gaps, $k$. When one such link is found, we are certain that arc $i j$ should not be added to the graph and proceed with the rest of the graph construction.

This process is followed only when two links have non-overlapping feasibility windows. For the randomly created problem set 2 described in VI.A, the average percentage of link pairs with non-overlapping windows is about $17 \%$ of the total number of pairs regardless of the problem size; small enough to not increase the computational time of the graph construction significantly.

\section{Cycle avoidance}

Every time a new link is added to the schedule, we set a node flag equal to false. This way, at each step, an ant will only consider the arcs leading to links that have not already been added to the schedule. The flags are all reset to true when one ant has finished with the path evaluation.

\section{Algorithms for comparison}

\begin{abstract}
A. Greedy
Greedy algorithms are usually of low computational complexity and simple to implement, thus can give feasible solutions fast; solutions that can, nevertheless, be far from optimal. We choose to compare our system with a greedy algorithm in order to confirm that the cost of designing a more sophisticated method is worth the effort, since it can perform significantly better in very complex problems. The performance increase can be translated to better usage of
\end{abstract}




\section{B. Squeaky Wheel Optimization}

In 1999, Joslin and Clements introduced the Squeaky Wheel Optimization (SWO) method [7]. In SWO, a solution is initially constructed by a greedy algorithm, and is then evaluated and analysed so that the "trouble" elements will appear. Those elements, if adjusted, are likely to improve the objective function value. After the appropriate changes take place, the greedy algorithm constructs a new solution; this continues while a certain criterion is not met. We have chosen this criterion to be the progress of the solution; if it is not becoming better within 15 iterations, then the search is restarted from a new initial condition. The search stops when the total number of available objective function evaluations is reached. SWO thus, is an algorithm iterating among solution construction, evaluation/analysis and element reordering. A high level workflow of the algorithm is shown in Algorithm 3 . 


\section{Initial Conditions}

SWO follows a deterministic process in the sense that for given initial conditions, it outputs the same result; in order to explore different areas of the search space and increase the performance of the algorithm, the process begins a number of times, from different initial conditions/solutions. In this paper, the mechanism presented in [21] for the production of the initial conditions is used. The tasks are initially ordered based on a criterion, the heuristic. In this application we break ties based on the e.s.t; the link with the smaller e.s.t. is placed higher in the order and the scheduler produces a feasible schedule based on this ordering. New initial conditions are produced by performing 20 random swaps in the initial task ordering in the nextInitCond() method every time the current solution stops having the expected progress. The first initial condition is the first solution produced by the greedy scheduler, without any swaps. The random swaps that result in different initial conditions per run render the SWO a non deterministic algorithm as a whole.

\section{Scheduler}

The links are scheduled based on the current order at the earliest available time, respecting their time windows l.e.t. - e.s.t. and the minimum gap between each pair of links, $k$. If a link conflicts with another, the next in order is considered. The process is performed by the produceSchedule() function in Algorithm 3

\section{3. 'Trouble-makers' identification and reordering}

Every time a schedule is produced, the unscheduled links are considered to be the 'trouble-makers' (term borrowed by [21]). The measure of the trouble caused by each link defines the number of forward moves it will do in the current order of all the links. In general, the more trouble caused by a link, the more forward moves in the order. The trouble metric function depends on the problem objective. Thus, we have chosen to set both the initial order criterion and the trouble metric equal to the heuristic function $\eta$ used for ACO in Eq. (5), i.e. either Eq. (2) or Eq. (4), depending on the objective under investigation. The two functions linked to the solution analysis and element reordering in Algorithm 3 are troubleEval() and reorder() respectively.

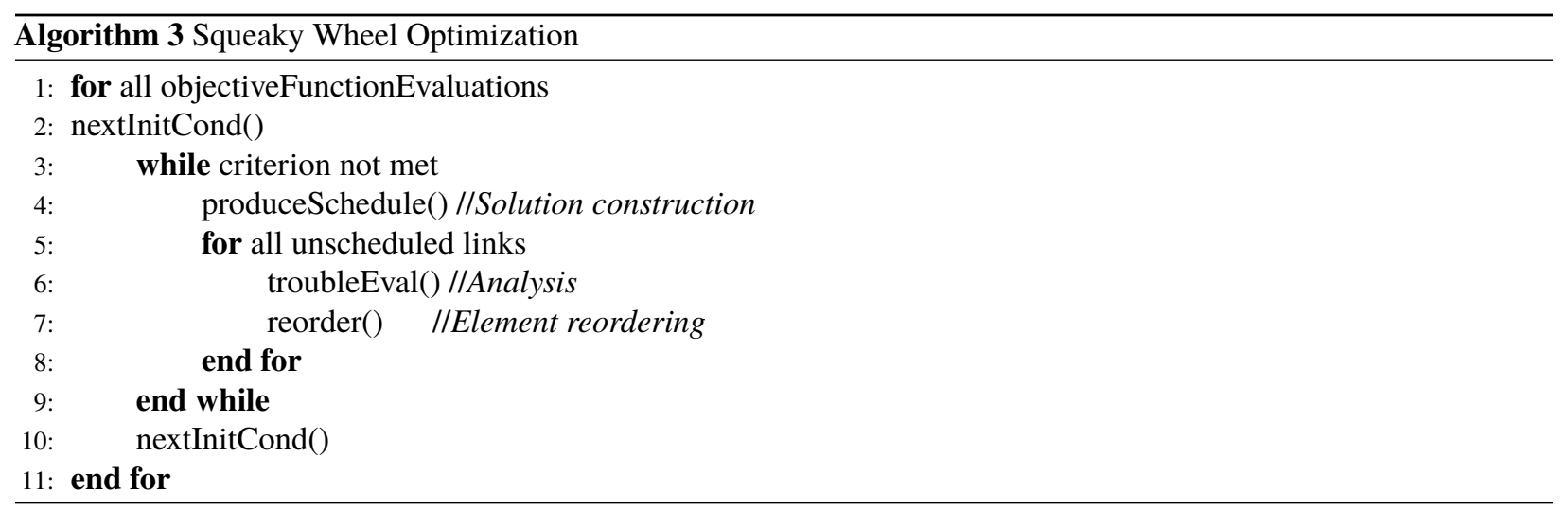



can be very short. Thus, one good metric of a MPS performance would not only involve the quality of the solutions it produces with respect to an optimum, but the quality of the solutions within the given amount of time. We measure the system efficiency by noting the median quality of the solutions it produces for different number of objective function evaluations.

- Scalability. The system performance should gracefully scale with the input size. In other words, an increase of the search space should not lead to large performance degradation. In order to investigate that aspect, we note the performance changes for an increasing problem size i.e. when 50, 100, 200 and 400 links arrive within a fixed planning horizon.

- $C P U$ or process time. The actual processing time needed from a Central Processing Unit (CPU) is measured in seconds and includes the total for each method:

- for ACO: the initial input read and graph construction and the path construction, evaluation and pheromone field update for all the ants

- for SWO: the initial input read and ordering and the schedule production, evaluation, trouble makers identification and the links' (re)ordering, for each step. The ordering algorithm used is Merge Sort [22] with $n \log (n)$ complexity.

In case of problem changes detected by the system, the graph environment is adjusted accordingly and the search is restarted. Both search restart and graph adjustment take place provided that the change is allowed to be taken into account i.e. before the users are informed about the time windows their links will be accommodated at. In that case we consider the following possible changes:

- Link deletion: the corresponding node is removed from the graph. In case this link has been identified as a connection between two links with non-overlapping windows from the pruning process described in Sect IV.C.2 those two links are connected with an arc of the proper direction.

- Changes in the feasibility windows (e.s.t, dur, l.e.t.) or new link addition: in such a case, the links' corresponding node connectivity in the graph is re-examined; Eq. (6) and 77 are used to check connectivity with all the other nodes of the graph anew.

- In case of link priority changes, the value of the corresponding node(s) is adjusted.

Ultimately, the percentage of the graph that will be adjusted depends on the size of the problem change. The search is restarted when a change occurs. Changes can occur after the links' start times have been communicated to the users, particularly the inability of a user to occupy the GEO resource on the scheduled time. In that case, 
the scheduled links are treated as idle time windows that break the initial planning horizon on shorter ones.

\section{A. Setting up the problems}

Given that we cannot access real data for data relay space missions, we create problems that are representative based on information collected from [23] and [24] regarding typical ranges for the planning horizon, the duration, start and end times and number of links. The problems are produced using uniform distribution within the ranges. Each problem consists of:

- A planning horizon of $m$ minutes.

- $G$ GEO resources.

- $N$ links $\in \mathcal{P}$, where $\mathcal{P}$ is the initial set of all the links, with

- Duration $(d u r) \in\left[15, \frac{m}{6}\right]$ minutes. The maximum value is constrained to $\frac{m}{6}$ in order to not have very long links and extreme solutions that consist of 1 or 2 links.

- A list of decreasing preference of the resources the link can be accommodated by, with the corresponding:

* Earliest start time (e.s.t.) in the set $[0, m-d u r]$ minutes.

* Latest end time (l.e.t.) in the set [e.s.t. + dur, $m)$ minutes.

- A priority value $p \in(0,1]$, with 0,1 indicating the lowest and highest priorities respectively.

- A minimum gap equal to $k$ minutes between each two consecutively scheduled links.

- An objective function $f$.

The dur, e.s.t. and l.e.t. values are produced randomly with uniform distributions within the given ranges.

In order to evaluate all three methods, we need to build a problem set that consists of problems of different difficulty so that we can distinguish among the algorithms. We begin by creating a problem set of 100 different problems in which we fix the best solution and note the divergence of each algorithm from it, in 100 independent runs. For this test, we consider a single GEO spacecraft. Objective (3) is considered, hence the heuristic used is (4). The links that belong to the best solution will have a maximum value of the heuristic function (4) and will all be able to be scheduled within the planning horizon that we set equal to 1 day or 1440 minutes. The maximum link priority value is 1 and the minimum duration time is 15 minutes. In order to calculate the number of links the optimal solution can consist of, we set every $k$ equal to the minimum gap between the links, which is 1 . The number of links in the best solution will be $\frac{1440}{15+k}=\frac{1440}{16}=90$. The maximum objective function value is equal to

$$
\max \{f\}=\frac{90 * 1}{\text { sum of all the links' priorities }}
$$

We consider the links to be as flexible as possible with e.s.t. $=0$ and l.e.t. $=1439$ thus increasing the options for their start times and, intuitively, the difficulty of the problem. We increase the level of oversubscription by adding another 


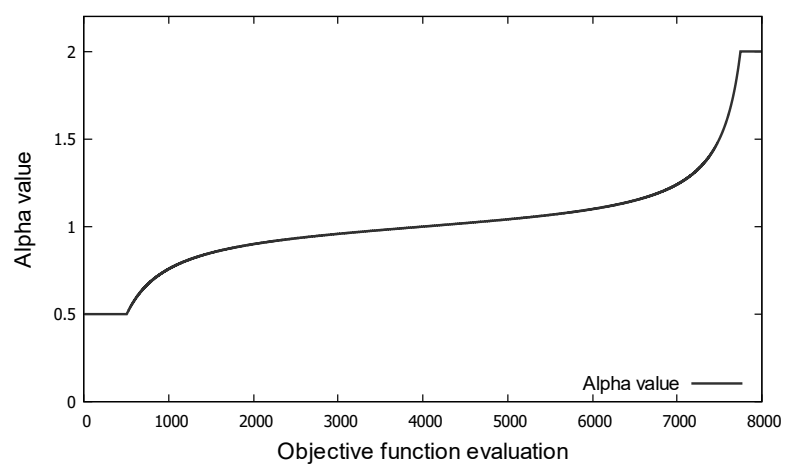

Fig. 3 Change of the alpha value within a run, for 8000 objective function evaluations.

110 randomly produced links and have a total of 200. The rest of the 100 different problems of the set are produced by adding a different set of 110 randomly produced links to the 90 that belong to the best solution; this is Problem Set 1 . We run ACO and SWO with 8000 objective function evaluations, $\beta=2$ in (5) and alpha $=g$ (evaluation step), where $g$ is an increasing function of the evaluation step. An example of $g$ for 8000 evaluations is shown in Figure 3 . For the initial conditions of SWO described in V.B.1, we choose $i c=400$ and $s=20$. In the reorder process, the links are assigned with $1-5$ forward moves, based on the trouble evaluation analysis. The algorithm has been written in Java, and the machine used is an Intel(R) Core(TM) i5-7200U CPU@ $2.50 \mathrm{GHz}$ processor running Windows 10.

In order to depict the results, we use box plots that include the minimum and maximum values of all the data, the median value is the straight line inside the box and $75 \%$ of the values are within the box whereas the range of the rest $25 \%$ is depicted with the whiskers. Any data not included between the whiskers is plotted as an outlier with a dot. In Fig. 4 we use the box plots for the 3 methods when Problem Set 1 is the input to the system, and run the problems 100 independent times. The Greedy method always finds the best solution as the straight line in value 1 denotes. SWO and ACO almost always do, but also have some outliers, probably due to their stochastic nature. Given this result, we assume that Problem Set 1 does not consist of problems that can help distinguish among the 3 methods and proceed to produce another set.

The new set (Problem Set 2) will consist of problems with 200 all of their links randomly produced, as described in the beginning of this section. Since we cannot know the value of the best solution for this set, the outcome of the methods is reduced to the best found value across all the runs and methods for each problem. Thus, the best value for all problems will remain equal to 1 . Figure $4 \mathrm{~b}$ shows the output for all the 3 methods; we now see different results. Not only the 3 algorithms perform differently, but there is also a wide spread among the solutions of Greedy and SWO; ACO most commonly produces the best result among the 3, hence the small spread in its solutions. The scaling between Fig. $4 \mathrm{a}$ and $4 \mathrm{~b}$ is different for demonstration purposes. The difference between the Greedy and the other two methods can be an indication that the problem difficulty increases.

Since problem set 2 leads to more interesting results, we continue the system testing with problem sets of different 
Problem Set 1

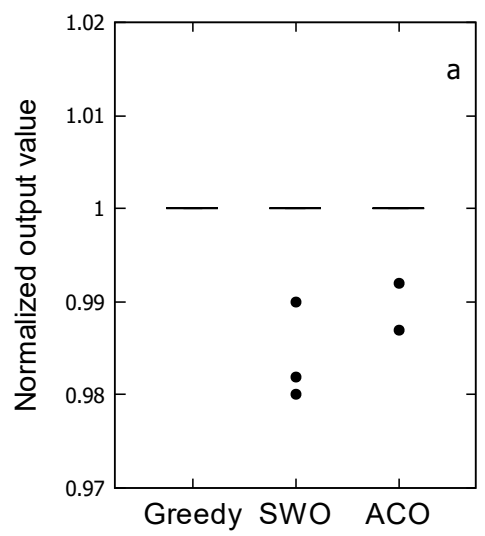

Problem Set 2

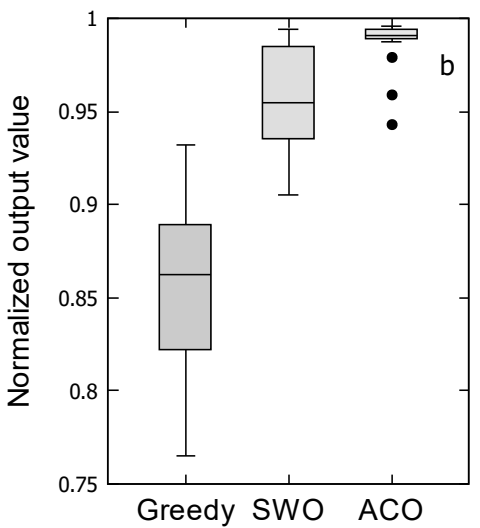

Fig. 4 Comparison of the 3 methods in problems of different difficulty

sizes produced in the same way. For the rest of the paper, Problem Sets 1 to 4 will now refer to sets of 100 different problems with 50, 100, 200 and 400 links respectively. Our tests begin with a single GEO resource being considered and in Sect. VI.E we discuss the constellation scenario.

\section{B. Efficiency Test}

The efficiency test contains the comparison of SWO and ACO with respect to the quality of their output for the available objective function evaluations they have. We expect that, for a fixed problem size, when the number of evaluations increases, the output value for both SWO and ACO will increase as well since more candidate solutions can be evaluated. Hence, both the median and best found values are generally expected to be larger. With this test, we aim at finding whether there is a number of evaluations above which the performance of the algorithms does not increase a lot, for a fixed problem size. Then, the ratio solution quality to number of evaluations will define the most efficient method. For example, in case of very similar solution quality, the method that uses fewer evaluations can be considered more efficient, due to the more targeted use of the available evaluations. In Fig. 5 and 6 we show the median output value of all the problems in each problem set for the 3 methods when the number of available evaluations increases from 500 to 8000, for both objectives (1) and (3). For the cases of 50 and 100 links in both figures, we also tested the system for 5 and 10 evaluations respectively, since those where the values at which ACO performed differently than in the rest of the evaluations' range $[500,8000]$. We plot the output of the greedy algorithm too, showing the performance difference from both SWO and ACO, regardless of the lack of iterations on this method.

For both objectives, evaluations and problem size, ACO outperforms SWO with respect to the quality of the output solution. The output value difference between the 2 methods lies within the range $[1.8,7.7] \%$ of the best found values i.e. the ACO values. Also, towards the higher end of the evaluations' range, both methods have similar trends (either increasing or stable) apart from Fig. 5 b, thus maintaining their difference. Therefore, it is safe to assume that within the 
50 Links
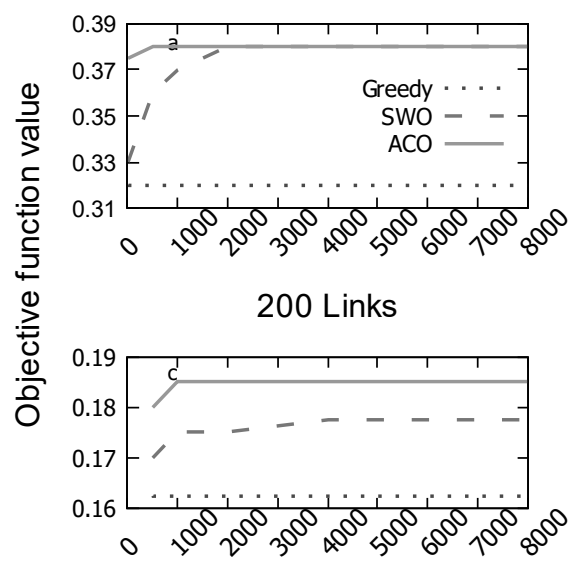

\# Objective function evaluations
100 Links
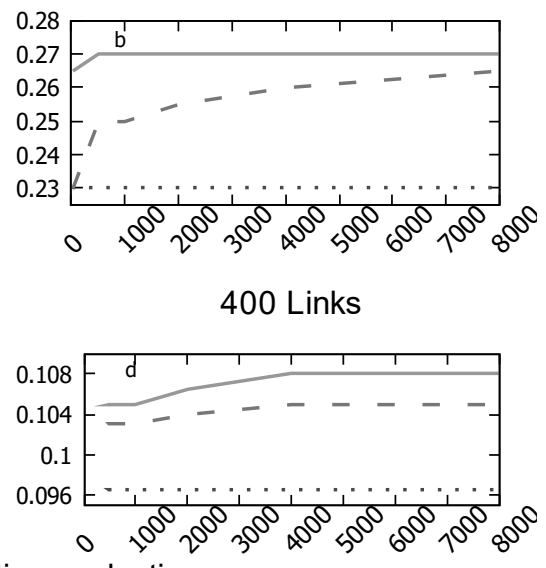

Fig. 5 Comparison of the 3 methods' efficiency for objective (1)

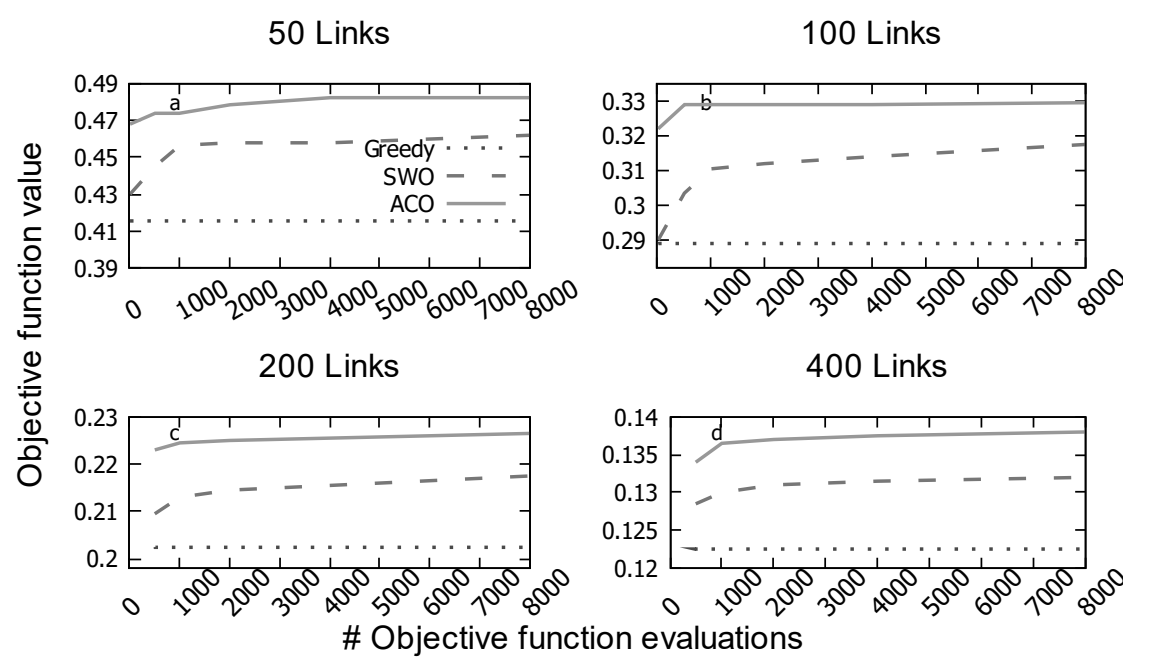

Fig. 6 Comparison of the 3 methods' efficiency for objective (3) 
range of objective function evaluations under investigation ([500, 8000]), ACO is more efficient than SWO.

For the sake of completeness of the comparison, we have implemented an exhaustive search algorithm to compute the optimal solution of the problems. Due to the high computational cost of computing the optimum for such large problems, we only apply the algorithm to the 50-link problems. In the exhaustive search algorithm, we produce all the possible link orders and each candidate solution is constructed by scheduling the links based on one order. If a link cannot be scheduled after other already scheduled ones, then the next in order is considered. The solution is complete when there aren't any links left to consider. The candidate solutions are then evaluated by the objective function and the optimal one is found.

Applying this method to the problem with objective function Eq. 11, the result is 0.38, which is equal to the value that both methods find for over 2000 evaluations. When we choose objective function Eq. 3, the output value is 0.485 , higher by $0.6 \%$ from the best found value by ACO and by $4 \%$ from the best found value by SWO.

\section{Scalability Test}

In this type of test we study how the problem size affects the algorithms' performance. An increasing problem size translates to more possible links' combinations which can lead to better solution quality. At the same time though, the search space becomes larger, which might hinder the performance. In this section, we first investigate whether the size increase results in solutions with better quality, and then study the rate by which the solution quality changes every time the problem size doubles. We expect the value of this rate to be decreasing as the problem size increases. The scalability of a method can be measured by how fast this rate decreases; the slower the decrease, the more scalable the algorithm is.

In Fig. $7 \mathrm{a}$ and $7 \mathrm{p}$ we note the performance of the 3 methods for both objectives in the 4 Sets problems, with $N=50,100,200,400$ links respectively. We run each problem 100 independent times, producing a median output value, and then a median solution value for all the problems in the same Problem Set is calculated and plotted in the figure. For the sake of brevity, we only show the result for 4000 objective function evaluations for both objectives, since when the rest numbers of evaluations are used (500, 1000, 2000 and 8000), the results are similar. This figure shows that the quality of the solutions found, from all 3 methods, with an increase in the problem size is decreasing. As this is contrary to what we expected, in Fig. 8 a and 80 we plot the unscaled objective functions i.e. the numerators' values for both objective functions. In this figure we see the expected increasing trend for all the methods. In other words, when the problem size increases, all 3 methods find schedules that consist of more links or have a higher sum of priorities. It is the increase in the value of the denominator of (1) and (3) that is larger that the corresponding increase in the numerator that leads to the behaviour noticed in Fig. 7 .

Fig. 8 clearly shows that the increase rate of the unscaled objective functions decreases for all methods as the problem becomes larger. Even though the rate difference among the 2 methods is not big enough to safely assume that one is more scalable than the other, the lines' trends indicate that for the given problem sizes ACO scales slightly better 
2

3

4

5

6

7

8
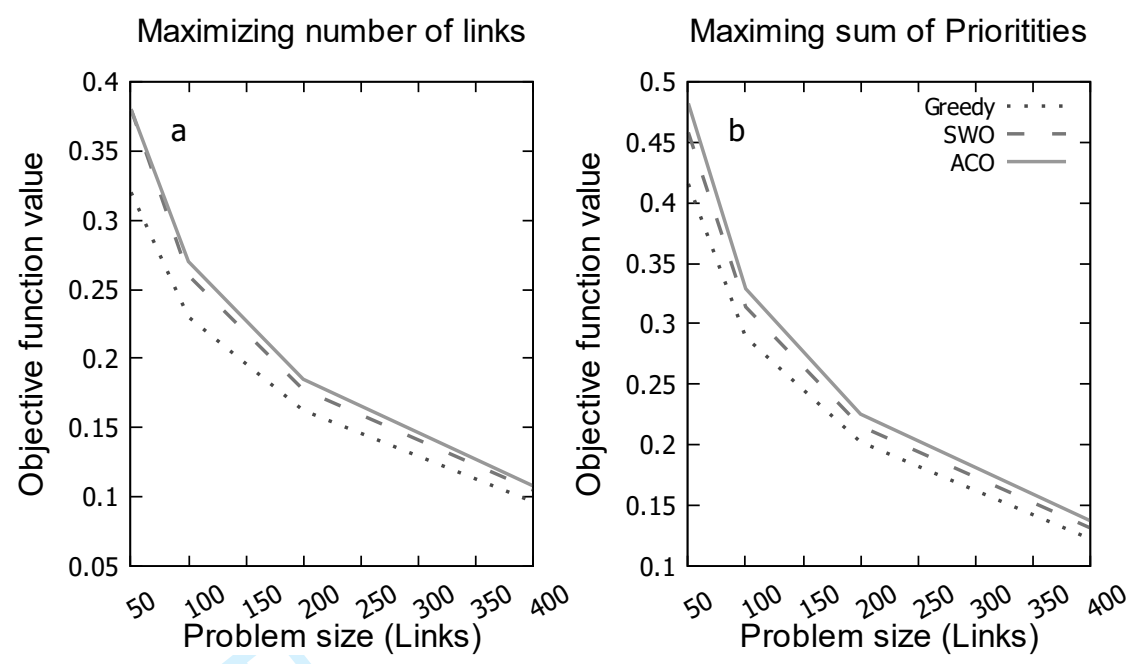

Fig. 7 Median output value per problem Set for 4000 evaluations.
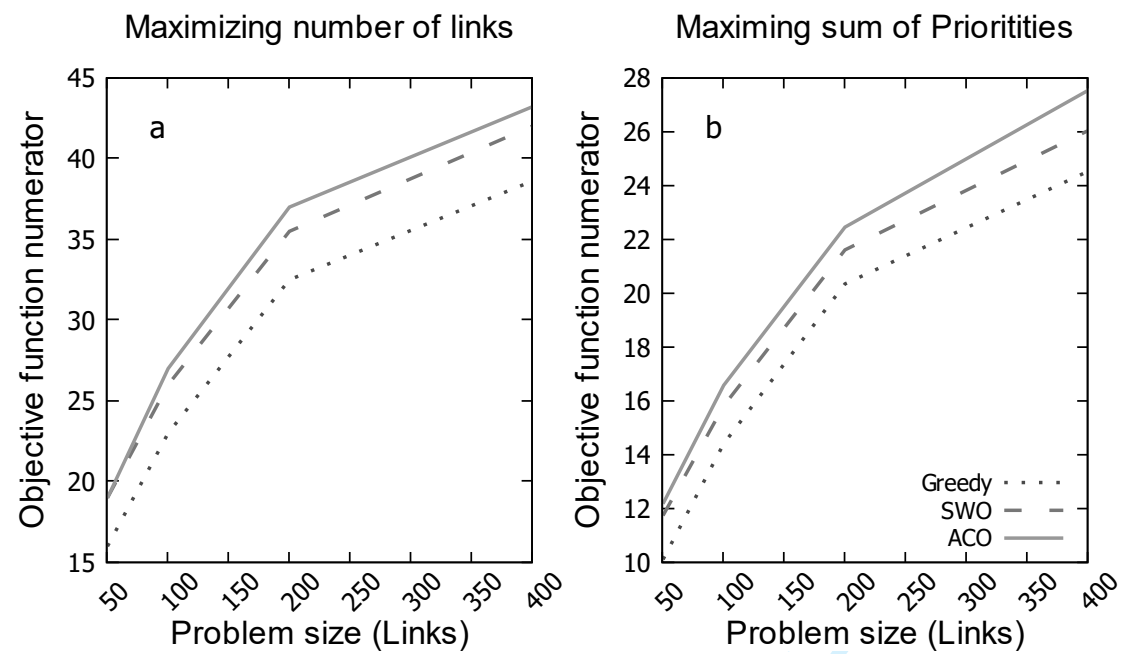

Fig. 8 Median numerator of the output value per problem Set for 4000 evaluations.

than SWO.

\section{CPU Time}

In this section, we investigate the effect the problem size and the number of evaluations have in the processing time (CPU Time). For the sake of brevity and to avoid showing similar results, when studying the effect of the problem size, we consider 4000 evaluations, and when studying the effect of the evaluations, we fix the problem size to 200 links. For the same reason, only objective (3) is studied. In Fig. 9a we show the median CPU time in Problem Set 3, for an increasing number of evaluations, and in Fig. 9p the median CPU time for the different problem sets and 4000 evaluations.

From both figures it is clear that ACO runs faster than SWO in all cases. This is due to the fact that the ants visit 

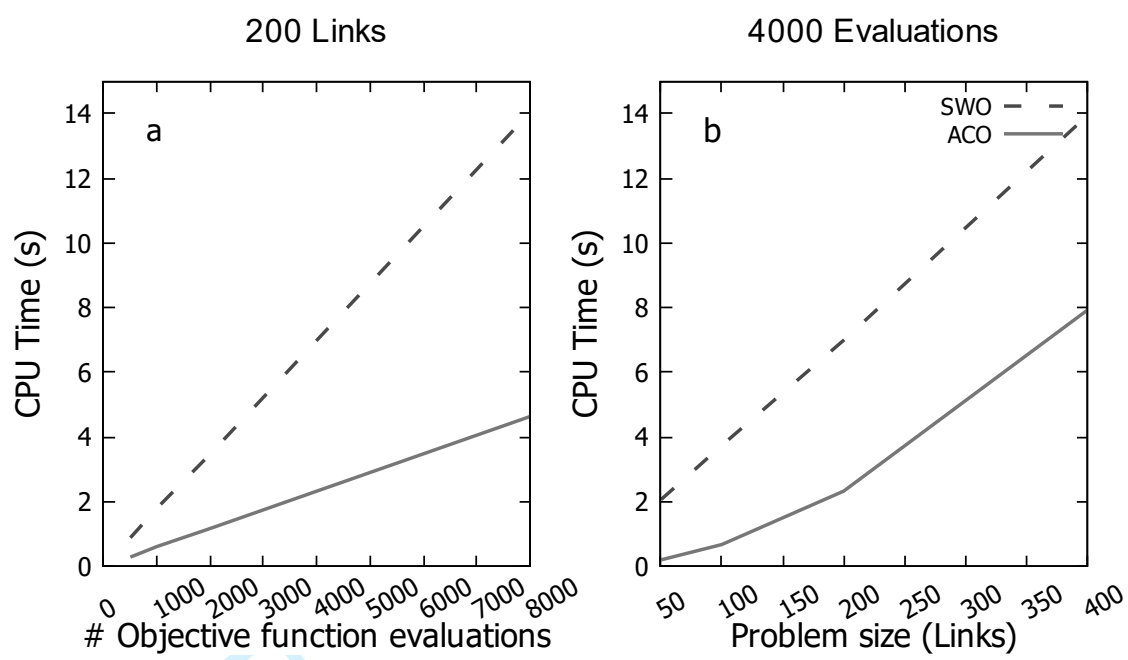

Fig. 9 (a)Median CPU Time per method for different number of evaluations when the input is Problem Set 3 and objective (3) (b)Median CPU Time per method for the different Problem Sets, 4000 evaluations and objective (3)

only 20 - 45 nodes on average (depending on the problem size) and do not traverse the whole graph, whereas in SWO all the links are evaluated per step, either by the objective function or by the trouble metric function. Naturally, we see that for both methods, the CPU time is doubled when the evaluations are doubled. When the problem size is doubled, CPU times scale linearly with the number of objective function evaluations for both methods but SWO requires more computational effort, regardless of the number of available evaluations. Most importantly, the two trends grow with a different rate, i.e. SWO CPU time increases $3 \%$ on average for each doubling of the input size, whereas the same rate for ACO is $1.5 \%$ on average. This characteristic can prove useful should the input size increase greatly. Another critical aspect is that the system's responsiveness capabilities are indirectly supported by the CPU time results; the computation time needed for a search is too short for a search restart in possible changes to be considered prohibiting.

\section{E. Constellation Scenario}

In Figures 10 and 11 we report the performance of SWO and ACO when multiple GEO resources are considered, and the median time a spacecraft is active, i.e. busy accommodating a link. The active time is measured from $0-1$ with 0 translating to the spacecraft being constantly idle, whereas 1 means that is it constantly active. The number of spacecraft is increased from 2 to 32, in a problem of 200 links to be scheduled within a day, when we are maximizing the number of links scheduled. With these graphs we want to conclude on the performance of the system, its scalability and its ability to balance the load among multiple spacecraft.

The information regarding the load balance among the spacecraft is included in the standard deviation of the median values of the active time. Our main observation is that the standard deviation in ACO is in general smaller than in SWO. This can translate to a better load balancing from the ACO algorithm. The fact that the median active time drops 
50 Links

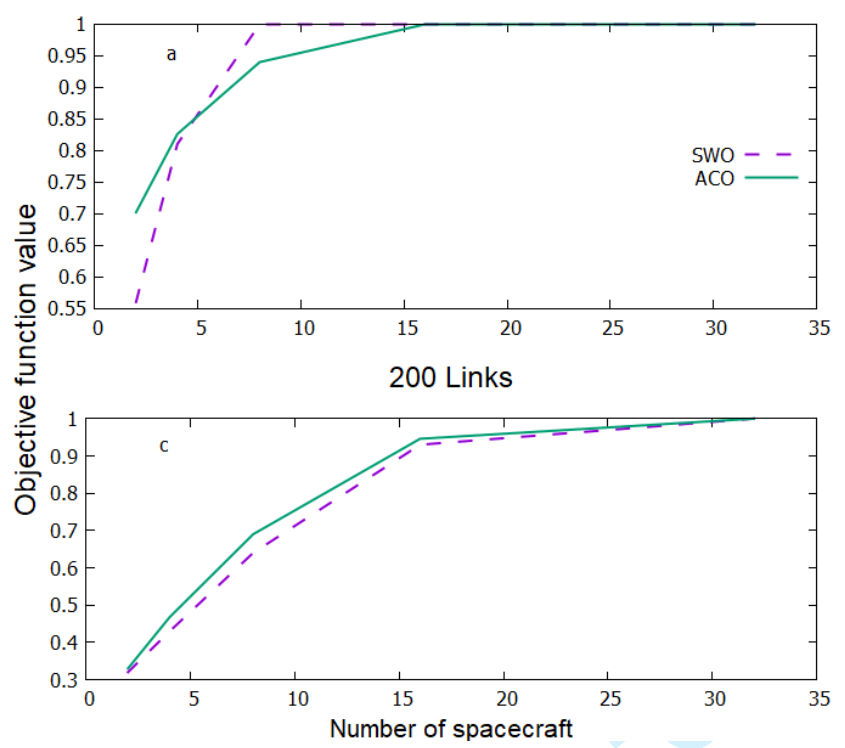

100 Links
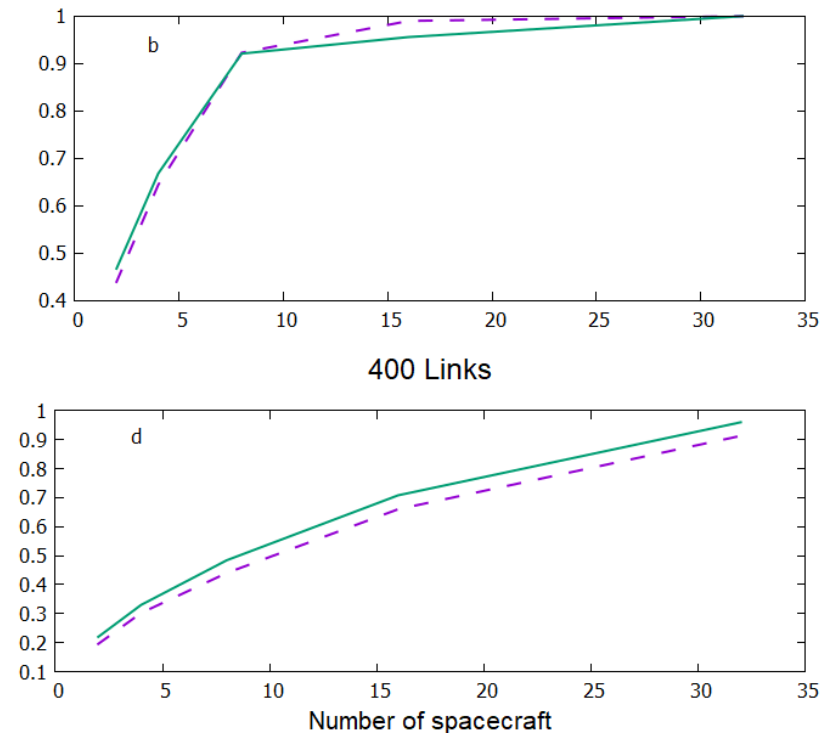

Fig. 10 Performance of SWO and ACO for an increasing number of spacecraft and (a) 50 (b) 100 (c) 200 and (d) 400 links, for objective 3 .
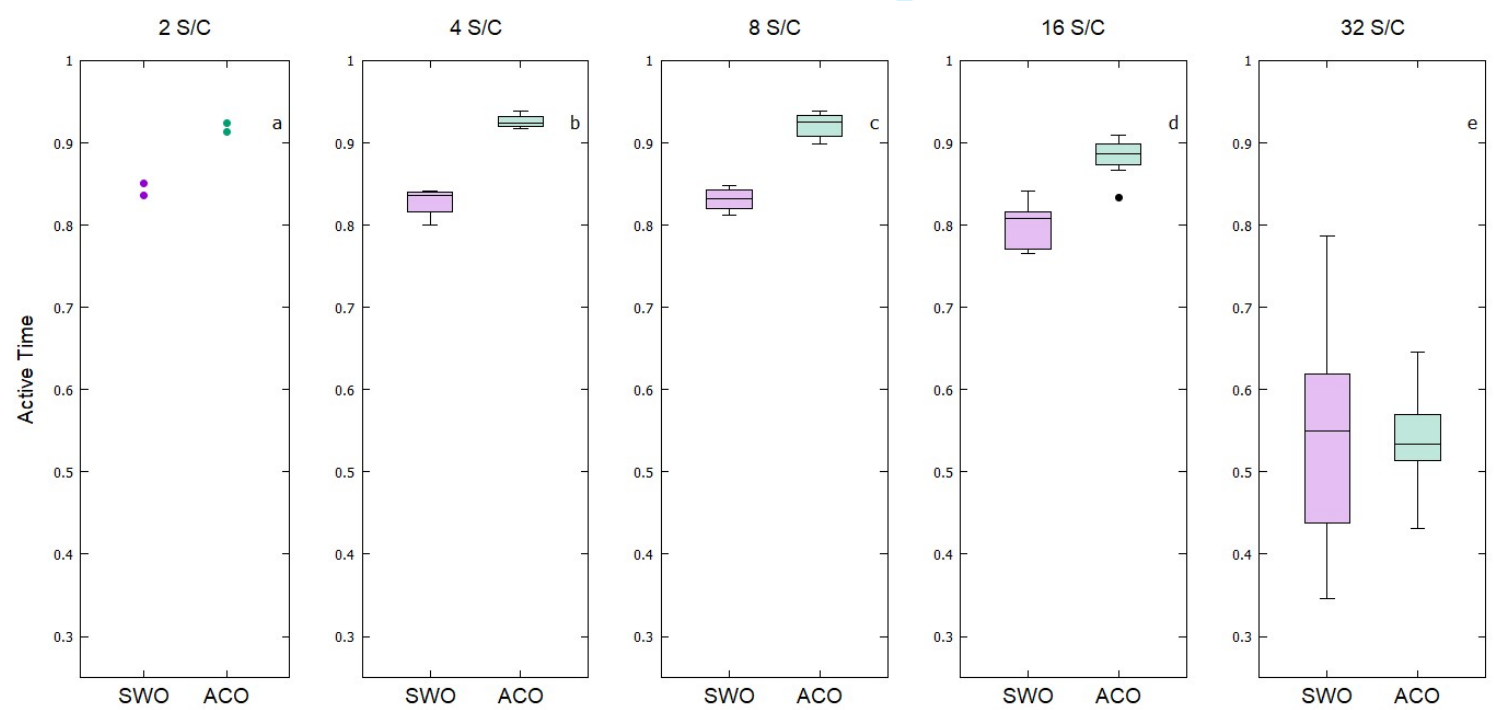

Fig. 11 Median active time per GEO spacecraft in a problem of 200 links with request to be scheduled within a planning horizon of 1 day. 
by a lot when 32 spacecraft are considered, reflects the fact that the problem has stopped being oversubscribed; this observation is also made clear in Fig. 10, where both methods reach the value of 1, for 32 spacecraft. Recalling the way the problems are designed, the number of spacecraft for which the links are available is randomly chosen from 1 to the maximum number of available spacecraft. That means that the links are not able to be scheduled in all the spacecraft, and the graph environment of each spacecraft is different. The reason for this difference between the two methods regarding the load balance lies on the way the links are assigned to the spacecraft: SWO assigns to the most highest GEO resource in the preference order associated with each link whereas ACO allows for the spacecraft to choose a link at each choice point.

\section{Conclusions and Future Work}

In this paper, we presented the oversubscribed scheduling problem that occurs in data relay space missions, where users submit more requests than can actually be scheduled. The difficulty of the problem lies in not only finding an optimal order for the requests, but first deciding on which requests will be scheduled, due to the lack of enough resources for all of them. The solving approach we proposed is based on the ants' foraging mechanism. In the real world, ants have the ability to find the shortest paths between their nest and a food source, communicating indirectly with each other, via the environment. This work describes the translation of the oversubscribed scheduling problem from the space operations' point of view to the ants' environment, and the application of an Ant Colony Optimization algorithm to find solutions. In the ACO algorithm presented, the ants combine heuristic information along with pheromone information to make a choice. The weight of each type of information changes during an algorithm run, since we have employed a dynamical exploration and exploitation balance. The system is tested on its efficiency i.e. how good are the solutions produced with a given number of objective function evaluations, its scalability to problems of larger size and its computational time. The comparison with Squeaky Wheel Optimization on problems with 50 - 400 flexible requests to be scheduled within one day shows that ACO is more efficient in finding good solutions and runs in less time than SWO, allowing for a fast replanning process in case of problem changes. Both methods are found to be almost equally scalable. When increasing the number of spacecraft from $2-32$, we noticed that the more oversubscribed the problem, the more steadily ACO outperforms SWO. An even more interesting finding though, is that, in addition to the optimized performance, ACO achieves better load balance among the spacecraft. This is achieved by using a simple random order of the spacecraft 'choosing' a link at each step, instead of assigning links to them.

To the best of the authors' knowledge, this is the first time ACO is applied to an oversubscribed scheduling problem. We address this class of problems by introducing additional features to a typical ACO algorithm:

- First node choice: given that the objective functions under investigation do not favour idle resource time in the solution, we choose the first node among the links with smaller earliest start times.

- Path completion criterion: Given that the problem is oversubscribed, only a subset of the nodes will be visited. 


\section{Acknowledgment}

This work is co-funded by the Surrey Space Centre (SSC) of the University of Surrey, the Surrey Satellite Technology Ltd (SSTL) and the Operations Centre of the European Space Agency (ESA/ESOC). 


\section{References}

[1] Gramling, J., and Chrissotimos, N., "Three Generations of NASA’s Tracking and Data Relay Satellite System,” SpaceOps 2008, Heidelberg, Germany, 2008.

[2] Wallrapp, F., Ballweg, R., and Gataullin, Y., “The European Data Relay System (EDRS): Operational Challenges,” 62nd International Astronautical Congress, Cape Town, SA, 2011.

[3] Donati, A., Policella, N., Rabenau, E., Righini, G., and Tresoldi, E., "An Automatic Planning and Scheduling System for the Mars Express Uplink Scheduling Problem," IEEE Transactions on Systems, Man, and Cybernetics, Part C (Applications and Reviews), Vol. 41, 2011, pp. 942-954. doi:https://doi.org/10.1109/TSMCC.2011.2114880.

[4] Johnston, M. D., and Giuliano, M., "Multi-Objective Scheduling for the Cluster II Constellation," 6th International Workshop on Planning and Scheduling in Space, 2011.

[5] Iacopino, C., Palmer, P., Brewer, A., Policella, N., and Donati, A., "EO Constellation MPS Based on ant Colony Optimization Algorithms," 6th International Conference on Recent Advances in Space Technologies - RAST2013, IEEE Press, Piscataway, NJ, 2013, pp. 159-164.

[6] Dorigo, M., and Stutzle, T., Ant Colony Optimization, MIT Press, 2004.

[7] Joslin, D., and Clements, D., “Squeaky Wheel Optimization,” Journal of Artificial Intelligence Research, Vol. 10, 1999, pp. 353-373. doi:https://doi.org/10.1613/jair.561.

[8] Neto, R. F. T., and Filho, M. G., "Literature review regarding Ant Colony Optimization applied to scheduling problems: Guidelines for implementation and directions for future research," Engineering Applications of Artificial Intelligence, Vol. 26, 2013, pp. 150-161. doi:https://doi.org/10.1016/j.engappai.2012.03.011.

[9] Mohan, B. C., and Baskaran, R., "A survey: Ant Colony Optimization based recent research and implementation on several engineering domain," Expert Systems with Applications, Vol. 39, 2012, pp. 4618-4627. doi:https://doi.org/10.1016/j.eswa.2011. 09.076 .

[10] Merkle, D., Middendorf, M., and Schmeck, H., "Ant Colony Optimization for Resource-Constrained Project Scheduling," IEEE Transactions on Evolutionary computation, Vol. 6, 2002. doi:https://doi.org/10.1109/TEVC.2002.802450.

[11] Huang, R.-H., and Yang, C.-L., "Ant colony system for job shop scheduling with time windows," The International Journal of Advanced Manufacturing Technology, Vol. 39, 2008, pp. 151-157. doi:https://doi.org/10.1007/s00170-007-1203-9.

[12] Manne, A. S., “On the Job-Shop Scheduling Problem,” Operations Research, Vol. 8, 1960, pp. 219-223. doi:https: //doi.org/10.1287/opre.8.2.219.

[13] Kramer, L. A., and Smith, S. F., "Maximizing flexibility: a retraction heuristic for oversubscribed scheduling problems," Proceedings of the 18th International Joint Conference on Artificial Intelligence, 2003, pp. 1218-1223. 
[14] Maillard, A., Verfaillie, G., and Pralet, C., “Adaptable Data Download Schedules for Agile Earth- Observing Satellites,” Journal of Aerospace Information Systems, Vol. 13, 2016, pp. 280-300. doi:https://doi.org/10.2514/1.I010383.

[15] Shouraboura, C., Johnston, M. D., and Tran, D., "Prioritization and Oversubscribed Scheduling for NASA's Deep Space Network,” Scheduling and Planning Applications Workshop - ICAPS 2016, London, UK, 2016.

[16] Troesch, M., Chien, S., and Ferguson, E., "Using Automated Scheduling to Assess Coverage for Europa Clipper and JUpiter ICy moons Explorer,” International Workshop on Planning and Scheduling for Space (IWPSS 2017), Pittsburgh, PA, 2017.

[17] Barbulescu, L., Howe, A., Whitley, L., and Roberts, M., "Understanding Algorithm Performance on an Oversubscribed Scheduling Application,” Journal of Artificial Intelligence Research, 2006, pp. 577-615. doi:http://dx.doi.org/10.1613/jair.2038.

[18] Eiben, A. E., and Schippers, C. A., “On evolutionary exploration and exploitation,” Journal Fundamenta Informaticae, Vol. 35, 1998, pp. 35-50. doi:http://dx.doi.org/10.3233/FI-1998-35123403.

[19] Iacopino, C., and Palmer, P., “The Dynamics of Ant Colony Optimization Algorithms Applied to Binary Chains,” Swarm Intelligence, Vol. 10, 2012, pp. 343-377. doi:https://doi.org/10.1007/s11721-012-0074-3.

[20] Meyer, B., "Convergence control in ACO," Genetic and Evolutionary Computation Conference (GECCO), Seattle, WA, 2004.

[21] Barbulescu, L., Whitley, L., and Howe, A. E., "Leap before you look: an effective strategy in an oversubscribed scheduling problem," AAAI'04 Proceedings of the 19th national conference on Artifical intelligence, San Jose, California, 2004, pp. $143-148$.

[22] Cole, R., "Parallel Merge Sort," SIAM Journal on Computing, Vol. 17(4), 1986, p. 770-785. doi:https://doi.org/10.1137/0217049.

[23] Barbulescu, L., Watson, J., Whitley, D., and Howe, A., "Scheduling space-ground communications for the Air Force satellite control network," Journal of Scheduling, Vol. 7(1), 2004, pp. 7-34. doi:http://dx.doi.org/10.1023/B:JOSH.0000013053.32600.3c.

[24] Scharringhausen, J.-C., and Beck, D. T., "Automated Procedure Based Operations for the European Data Relay System,” 68th International Astronautical Congress (IAC), Adelaide, Australia, 25-29 September 2017.

[25] Tripp, H., and Palmer, P., “Stigmergy for Nanosatellite Cluster Coordination without Intersatellite Links,” Vol. 8, 2011, pp. 127-150. doi:http://dx.doi.org/10.2514/1.48212. 